\title{
Chronic heart failure in the elderly: value of cardiopulmonary exercise testing in risk stratification
}

\author{
L C Davies, D P Francis, M Piepoli, A C Scott, P Ponikowski, A J S Coats
}

\begin{abstract}
Objective-To assess the value of cardiopulmonary exercise testing in predicting prognosis in a cohort of elderly patients with chronic heart failure (CHF).

Design-A retrospective cohort study of all patients with CHF over the age of 70 years assessed between January 1992 and May 1997.

Setting-Tertiary centre.

Patients-50 patients (mean (SD) age 75.9 (4.5) years, 8 women) with CHF New York Heart Association (NYHA) class I (3 patients), II (25 patients), III (20 patients), and IV (2 patients). Follow up was complete for two years in all patients.

Results-The patients underwent cardiopulmonary exercise testing (peak oxygen consumption $15.2(4.5) \mathrm{ml} / \mathrm{kg} / \mathrm{min}$, minute ventilation/carbon dioxide production $\left(\mathrm{VE} / \mathrm{VCO}_{2}\right)$ slope 38.7 (11.8)); radionucleide ventriculography (left ventricular ejection fraction 32.8 (14.3)\%); serum sodium measurement (139 (2.8) mmol/l); and echocardiography (left ventricular end diastolic dimension $6.1(1.1) \mathrm{cm}$, left ventricular end systolic dimension $4.7(1.5) \mathrm{cm})$. At the end of follow up in May 1999, 26 patients had died. The median follow up of the survivors was 47.7 months (interquartile range 31.5-53.5 months). On univariate analysis $\mathrm{VE} / \mathrm{VCO}_{2}$ slope $(\mathrm{p}<0.0001)$, NYHA class $(\mathrm{p}<0.001)$, peak oxygen uptake $\left(\mathrm{VO}_{2}\right)(\mathrm{p}<0.01)$, left ventricular end systolic dimension $(\mathrm{p}<0.05)$, and serum sodium concentration $(\mathrm{p}<0.05)$ had significant predictive power. Stepwise multivariate analysis identified only VE/ $/ \mathrm{VCO}_{2}$ slope $(\mathrm{p}<0.01)$, NYHA class $(\mathrm{p}<0.05)$, and peak $\mathrm{VO}_{2}(\mathrm{p}<0.05)$ as conveying significant independent prognostic information.

Conclusion-Elderly patients with CHF have a high mortality, with the majority dead within two years. Cardiopulmonary exercise testing provides important information for risk stratification within this group and its use should not be neglected.
\end{abstract}

(Heart 2000;83:147-151)

Keywords: cardiopulmonary exercise testing; chronic heart failure; elderly patients; risk factor stratification

Chronic heart failure $(\mathrm{CHF})$ is a common condition especially among the elderly, ${ }^{1}$ with a prevalence of $10 \%$ above the age of 75 years. $^{2}$ It not only causes significant mortality but also carries substantial morbidity, and is the underlying or principal reason for many hospital admissions. ${ }^{3}$ Prognostic studies in younger patients have shown the usefulness of cardiopulmonary exercise testing in heart failure assessment, particularly for the selection of those who may benefit from cardiac transplantation. ${ }^{4}$ It is not known, however, whether cardiopulmonary exercise testing has a comparable role in risk stratification of elderly patients with CHF.

We therefore set out to study the prognosis of CHF patients over 70 years old and to determine which of the clinical variables measured routinely was best at predicting survival.

\section{Methods}

SUBJECTS

A cohort of outpatients over 70 years old with stable $\mathrm{CHF}$ was identified who had undergone assessment at a tertiary referral centre between January 1992 and May 1997. Patients were included on the basis of clinical assessment (a history of dyspnoea and symptomatic exercise intolerance with signs of pulmonary congestion or peripheral oedema) and/or evidence of impaired left ventricular function from radionucleide ventriculography or echocardiography.

All patients underwent symptom limited cardiopulmonary exercise testing on a motorised treadmill. A modified Bruce protocol ${ }^{5}$ was used with an additional "stage 0 " ( $3 \mathrm{~min}$, speed $1 \mathrm{mph}, 5 \%$ gradient). Minute ventilation (VE), oxygen uptake $\left(\mathrm{VO}_{2}\right)$, and carbon dioxide production $\left(\mathrm{VCO}_{2}\right)$ were monitored continuously using a respiratory mass spectrometer (Amis 2000, Innovision, Odense, Denmark). Patients were encouraged by the supervising physician to exercise to the limit of their symptoms. All but six patients achieved a respiratory quotient of more than 1.00, indicating the achievement of anaerobic exercise conditions (mean (SD) 1.09 (0.12)). The $\mathrm{VE} / \mathrm{VCO}_{2}$ slope, which relates the rate of increase in ventilation per unit increase in carbon dioxide production, was obtained by linear regression analysis. ${ }^{6}$

Survival was determined by liaison with the UK Office of National Statistics which maintains a registry of survival for all of the subjects studied. No patient was lost to follow up. At least two years of follow up was available in all patients. 
STATISTICAL ANALYSIS

Statistical calculations were performed using the Statview 4.5 package (Abacus Concepts, Berkeley, California, USA). Numerical values are presented as mean (SD). The prognostic value of parameters considered as continuous variables were determined using the Cox proportional hazards linear regression model. A value of $\mathrm{p}<0.05$ was considered significant. Univariate analyses were conducted with several continuous variables and with New York Heart Association (NYHA) class dichotomised into I and II versus III and IV. A multivariate survival model was then constructed using the forward-backward method.

\section{Results}

PATIENT CHARACTERISTICS

Fifty patients were studied. The mean age at the time of exercise testing was 75.9 (4.5) years (range 70.2-86.1 years). Forty two patients were men and eight were women. Three of the patients were in NYHA class I, 25 in class II, 20 in class III, and two in class IV. The cause of chronic heart failure was ischaemic heart disease in 33 patients, idiopathic dilated cardiomyopathy in 13 patients, and valvar disease in two patients. Ischaemic heart disease was diagnosed by either cardiac catheterisation or a history of previous myocardial infarction or angina. Radionucleide ventriculography $(\mathrm{n}=37)$ showed an average left ventricular ejection fraction (LVEF) of 32.8 (14.3)\%. Echocardiographic quantification of left ventricular dimensions $(n=49)$ showed an end diastolic dimension (LVEDD) of $6.1(1.1) \mathrm{cm}$, and an end systolic dimension (LVESD) of 4.7 (1.5) $\mathrm{cm}$. The peak $\mathrm{VO}_{2}$ was $15.2(4.5) \mathrm{ml} / \mathrm{kg} /$ $\mathrm{min}$, and the $\mathrm{VE} / \mathrm{VCO}_{2}$ slope was 38.7 (11.8).
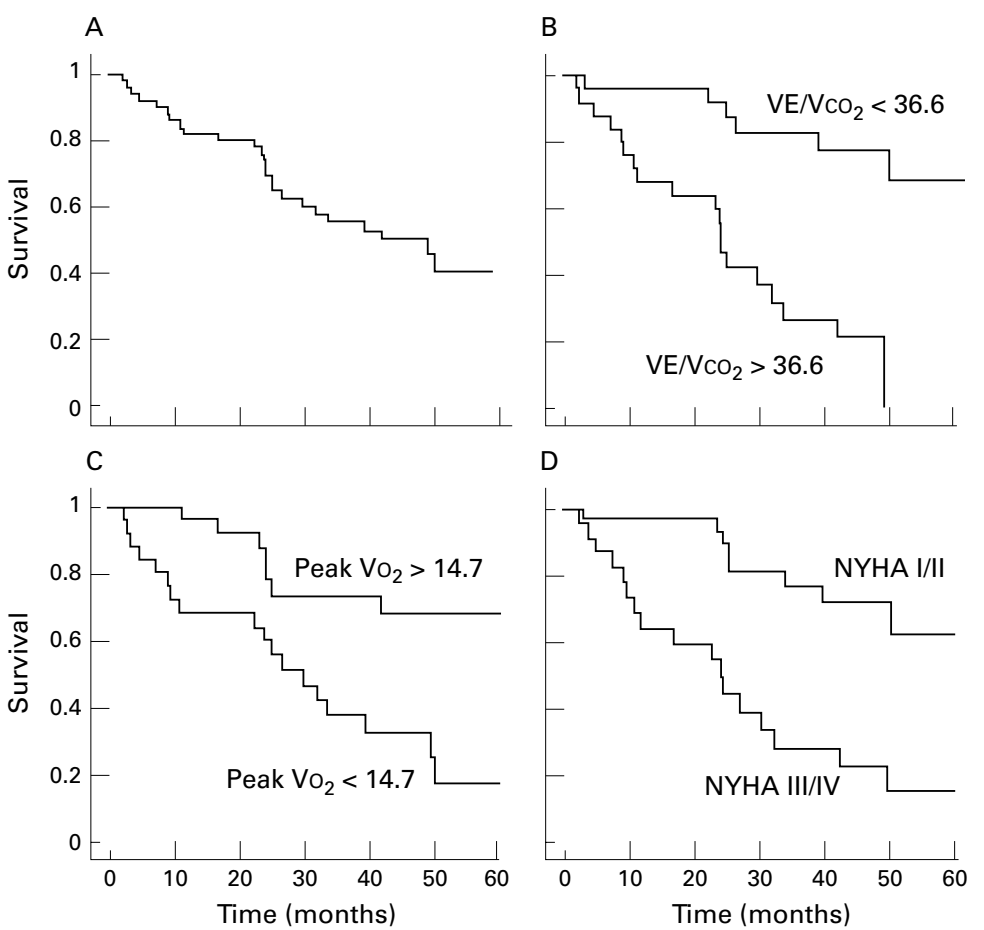

Figure 1 Kaplan-Meier survival curves for (A) total population, (B) $\mathrm{VE} / \mathrm{VCO}_{2}$ slope, (C) peak $V_{O_{2}}$, and (D) NYHA class I/II versus III/IV. The median values of peak $V_{O_{2}}$ and $\mathrm{VE} / \mathrm{VCO}_{2}$ were used to dichotomise the patients for the purpose of display in $(B)$ and $(C)$.
Table 1 Univariate Cox proportional hazards analysis of predictors of two year mortality

\begin{tabular}{|c|c|c|}
\hline Variable & $\chi^{2}$ & p Value \\
\hline VE/ $/ \mathrm{VCO}_{2}$ slope & 17.7 & $<0.0001$ \\
\hline NYHA & 12.6 & 0.0004 \\
\hline Peak $\mathrm{VO}_{2}$ & 11.1 & 0.0009 \\
\hline Serum sodium & 5.19 & 0.02 \\
\hline LVESD & 4.14 & 0.04 \\
\hline Weight & 2.44 & 0.12 \\
\hline Age & 2.27 & 0.13 \\
\hline Sex & 2.2 & 0.14 \\
\hline Ischaemic aetiology & 1.35 & 0.25 \\
\hline LVEDD & 0.81 & 0.37 \\
\hline LVEF & 0.56 & 0.46 \\
\hline
\end{tabular}

See text for explanation of abbreviations.

Table 2 Multivariate Cox proportional hazards analysis of predictors of two year mortality

\begin{tabular}{|c|c|c|}
\hline Variable & $\chi^{2}$ & p Value \\
\hline $\mathrm{VE} / \mathrm{VCO}_{2}$ slope & 7.02 & 0.008 \\
\hline NYHA & 6.57 & 0.01 \\
\hline Peak $\mathrm{VO}_{2}$ & 4.25 & 0.04 \\
\hline
\end{tabular}

The serum sodium concentration $(n=48)$ was $139(2.8) \mathrm{mmol} / \mathrm{l}$. At the time of exercise testing, $82 \%$ of patients were on an angiotensin converting enzyme (ACE) inhibitor or angiotensin II antagonist, $46 \%$ were on aspirin, $90 \%$ were taking a loop diuretic, $32 \%$ were on a thiazide diuretic, $14 \%$ were on a $\beta$ blocker, $21 \%$ were on a long acting nitrate preparation, $46 \%$ were taking digoxin, and $40 \%$ were on warfarin. Fourteen per cent of the patients had permanent pacemakers and $18 \%$ had chronic atrial fibrillation.

SURVIVAL

At the end of the follow up period in May 1999, 26 patients had died (median time to death 23.9 months, interquartile range (IQR) 9.2-31.8 months). The Kaplan-Meier survival curve is shown in fig $1 \mathrm{~A}$. The one year mortality was $18 \%$ and the two year mortality was $29 \%$. The median follow up duration for the survivors was 47.7 months (IQR 31.5-53.5 months). Deaths were not classified as being the result of progressive heart failure, sudden death, or non-cardiac as accurate information was not available.

On univariate analysis by the Cox proportional hazards method, the continuous peak $\mathrm{VO}_{2}$, $\mathrm{VE} / \mathrm{VCO}_{2}$ slope, serum sodium concentration, LVESD, and the dichotomous variable NYHA class (I/II versus III/IV) had significant predictive power at the $5 \%$ level. No significant predictive power was seen with the other variables tested including age, sex, weight, LVEF, LVEDD, or presence of ischaemic heart disease. These results are summarised in table 1 .

A multivariate survival model was developed by a stepwise process; this identified only $\mathrm{VE} / \mathrm{VCO}_{2}$ slope, NYHA class, and peak $\mathrm{VO}_{2}$ as conveying significant independent prognostic information (table 2). Kaplan-Meier survival plots were constructed to illustrate the prognostic significance for each of these three variables (fig 1B-D).

A variety of cut off values was applied to the continuous variables that were significant in the univariate analysis and also LVEDD and LVEF. The specificity and sensitivity (for 


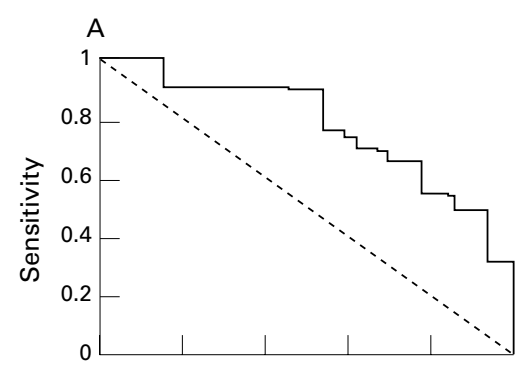

B
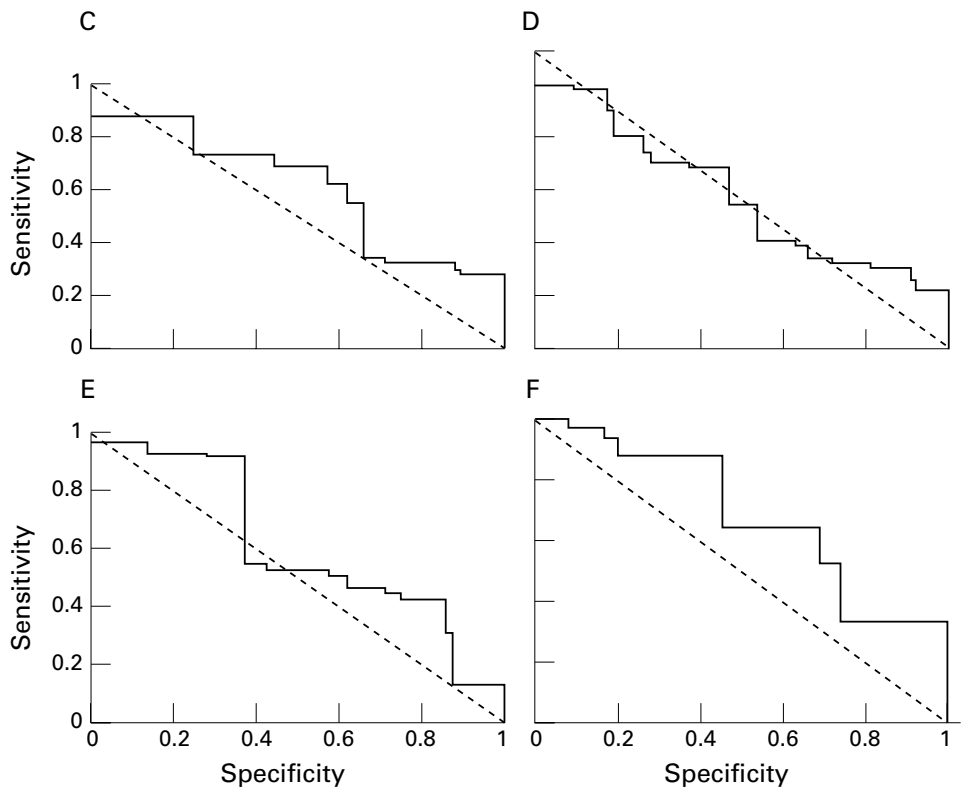

Figure 2 ROC curves for (A) peak $V_{O_{2}}$, (B) $V E / V_{C O}$, (C) LVESD, (D) LVEDD, (E) LVEF, and $(F)$ serum sodium concentration.

predicting mortality) at two years were assessed for each variable in the form of receiver operating characteristic (ROC) curves (fig 2). ${ }^{7}$ In the plots, the diagonal dotted line (the area under curve (AUC) of which is 0.5) represents a theoretical test with no prognostic value. A test that perfectly distinguishes survivors from those who will die within two years has an AUC of 1.0. Using this scale, the areas under each curve were 0.82 for $\mathrm{VE} / \mathrm{VCO}_{2}$ slope, 0.76 for peak $\mathrm{VO}_{2}, 0.64$ for LVESD, 0.56 for LVEDD, 0.54 for serum sodium concentration, and 0.58 for LVEF.

\section{Discussion}

The elderly with CHF are known to have a poor prognosis, ${ }^{38-10}$ but few studies have investigated prognostic variables among these patients. One study followed 399 patients over the age of 70 years hospitalised with CHF. ${ }^{11}$ Thirty four per cent of these patients had preserved left ventricular systolic function. Independent predictors of one year mortality included lower systolic blood pressure and activities of daily living score and higher blood urea and NYHA class. In those patients who had evidence of impaired left ventricular systolic function, the activities of daily living score, systolic blood pressure, and blood urea remained as independent predictors, but NYHA class was replaced by reduced serum albumin. LVEF, although predictive for readmission at three months, was not a predictor of one year mortality in either group of patients. A retrospective study of 94 men over the age of 75 years with $\mathrm{CHF}$ showed a high mortality when compared to age matched controls; once again, LVEF was not predictive of survival. ${ }^{12}$ Mortality rates can be as high as $63 \%$ in the very old (over 80 years) who are in long term institutionalised care. $^{8}$

No studies have assessed the prognostic value of cardiopulmonary exercise test parameters in elderly CHF patients, despite the fact that peak $\mathrm{VO}_{2}$ is widely used in younger age groups. ${ }^{4}$ It may be felt that since older subjects have lower exercise capacities, the discriminatory power of cardiopulmonary exercise testing would be reduced. It has been shown, however, that values obtained during cardiopulmonary testing (peak $\mathrm{VO}_{2}$ and ventilatory anaerobic threshold) have good reproducibility in elderly patients with $\mathrm{CHF}{ }^{13}$

We have confirmed that the mortality for patients with CHF over the age of 70 years is high, even in a population of stable outpatients. Individually, the $\mathrm{VE} / \mathrm{VCO}_{2}$ slope, peak $\mathrm{VO}_{2}$, NYHA class, LVESD, and serum sodium concentration gave useful prognostic information in this population. Age, sex, LVEF, LVEDD, and presence of ischaemic heart disease were not predictors of mortality. In multivariate analysis, the significant prognostic indicators were $\mathrm{VE} / \mathrm{VCO}_{2}$ slope, NYHA class, and peak $\mathrm{VO}_{2}$.

The strongest predictor of mortality in this study was the $\mathrm{VE} / \mathrm{VCO}_{2}$ slope, which measures the increase in ventilation to a unit increase in carbon dioxide production. It has been shown that the gradient of this slope is greater in patients with CHF when compared to normal controls ${ }^{14}$ and is associated with poorer exercise tolerance. ${ }^{15}$ Several abnormalities have been linked to this phenomenon, including ventilation/perfusion mismatch, ${ }^{16}$ abnormal pulmonary vascular haemodynamics, ${ }^{17}$ increased anatomical ${ }^{18}$ and physiological ${ }^{19}$ dead space, and possibly most importantly disordered ventilatory reflex control. ${ }^{20}$ The high predictive power of $\mathrm{VE} / \mathrm{VCO}_{2}$ may relate to the fact that it is computed from an average of a large amount of information and is therefore less susceptible to vagaries of $\mathrm{CHF}$ such as irregular breathing that may interfere with determination of peak $\mathrm{VO}_{2}$.

The importance of peak $\mathrm{VO}_{2}$ assessment in younger patients with $\mathrm{CHF}$ is well recognised, especially during assessment for possible cardiac transplantation. ${ }^{21} \mathrm{~A}$ range of cut off values has been proposed, ranging from 10-18, below which the prognosis is poor. ${ }^{41-25}$ We have now shown that peak $\mathrm{VO}_{2}$, when viewed as a continuous variable, is also a strong predictor of survival in elderly patients with CHF. Low values of peak $\mathrm{VO}_{2}$ can be caused by a variety of factors ${ }^{26}$ including limitation in cardiac output, poor peripheral blood flow, ${ }^{6}{ }^{27}$ impaired skeletal muscle metabolism, ${ }^{28}$ or early test termination because of cardiac related or other symptoms. The high prognostic value ${ }^{29}$ of peak $\mathrm{Vo}_{2}$ may result from the fact that it embodies a combination of many mechanisms of functional capacity, where impairment of each is a physiological disability. 
Functional assessment by the NYHA classification is widely used by clinicians to determine the severity of symptoms in patients with $\mathrm{CHF}^{30}$ Despite being subjective, poorly reproducible, and lacking sensitivity in reflecting changes in patient status, it has been shown to be a powerful predictor of mortality. ${ }^{22}$ The resilience of this simple clinical variable may again reflect its dependence on multiple important physiological factors.

This study also showed predictive value (in univariate analysis only) of serum sodium concentration and LVESD. Both these variables have previously been shown to predict mortality in patients with $\mathrm{CHF}^{31-33}$ Low serum sodium reflects activation of the reninangiotensin system $^{31}$ and possibly also increased diuretic use in patients with more severe fluid retention. ${ }^{34}$

Of some interest was the inability of LVEF (as measured by radionucleide ventriculography) in our study to predict mortality in patients with impaired left ventricular systolic function. Most previous studies have shown that LVEF, when assessed by radionucleide ventriculography, does predict mortality. ${ }^{10} \mathrm{Ex}-$ ceptions do exist, however, ${ }^{35}$ particularly in the elderly. ${ }^{12}$ It is now widely recognised that peripheral abnormalities such as activation of chemoreflexes, muscle ergoreceptors, neurohormonal and immune systems, and attenuation of baroreflex sensitivity play a very important role in symptoms and prognosis in $\mathrm{CHF}^{36}$ and may therefore account for the low predictive power of measures of pure ventricular function.

\section{STUDY LIMITATIONS}

The principal limitation in any study of the natural history of a disease which has a spectrum of severity is that its findings might only be valid for the segment of the spectrum that is being addressed. The overall frailty of a group of elderly subjects can be summarised by their mortality: a large difference in overall mortality between studies would imply populations of different disease severity. The illest patients are those requiring emergency admission to hospital for heart failure. Those that survive admission and the initial high risk period immediately after discharge may be considered to be outpatients with stable chronic heart failure. A large and prospective study from the USA addressing elderly patients (mean age 78 years) with systolic heart failure (mean LVEF 28\%) who were still alive three months after admission documented a subsequent mortality of $19.4 \%$ per year. ${ }^{11}$ Another prospective study from Sweden of elderly patients (mean age 76 years) with systolic heart failure (estimated mean LVEF 35\%) reported that those patients surviving one year after index admission had a mortality over the next two years of $36.6 \%$, although only $66 \%$ of patients were receiving ACE inhibitors (compared to $82 \%$ in our study). ${ }^{37}$ The Framingham heart study from the USA involved the prospective observation of a large number of individuals with an evaluation that included routine echocardiography. ${ }^{38}$ The annual mortality rate in those found to have the clinical syndrome of heart failure and systolic dysfunction on echocardiography was $18.9 \%$. Only $36 \%$ were on ACE inhibitors, which was typical of clinical practice in the late 1980 s. In our study, the Kaplan-Meier mortalities were 18\% at one year and $29 \%$ at two years, which suggests that our population is typical of general hospital outpatient populations of elderly patients with stable CHF. Our centre's chronic heart failure service receives direct referrals from general practitioners and from other hospitals, and uses cardiopulmonary exercise testing routinely in all patients with known or suspected heart failure. The exercise protocol has a gentle initial stage, which enables measurement of peak $\mathrm{VO}_{2}$ and $\mathrm{VE} / \mathrm{VCO}_{2}$ slope in any patient who can walk a few steps unaided. Advanced age and concomitant pathology are not themselves contraindications to exercise.

Patients with symptoms of heart failure but preserved systolic function were excluded from this study. Diastolic dysfunction, in which the ventricles may be small and thus the ejection fraction enhanced, may carry an adverse prognosis similar to that associated with left ventricular systolic dysfunction. ${ }^{9}{ }^{12}$ This would cause a $U$ shaped relation between mortality and ejection fraction, with high risk at both low and high values. In such circumstances, the common use of the single, raw ejection fraction as a parameter in a Cox survival model (or a threshold in a Kaplan-Meier model) must necessarily underestimate the prognostic information available from the variable. We speculate that because objective measures of exercise physiology such as peak $\mathrm{VO}_{2}$ and $\mathrm{VE} / \mathrm{VCO}_{2}$ slope (and subjective measures such as NYHA class) do not suffer from this limitation of paradoxical values with diastolic heart failure, they have the potential to apply to the full spectrum of heart failure in the elderly.

\section{CONCLUSIONS}

The prognosis for elderly patients with $\mathrm{CHF}$ is poor. Cardiopulmonary exercise testing provides a continuous and objective grading of $\mathrm{CHF}$ patients which is prognostically more useful than resting measures of ventricular function. This information is statistically additive to the NYHA classification which, despite being cheap and quick, is subjective and can describe only large changes in clinical status. We therefore suggest that cardiopulmonary exercise testing should be considered in the assessment of any patient with $\mathrm{CHF}$ regardless of age.

Dr Davies is supported by the Robert Luff Fellowship. Dr Francis is supported by the British Heart Foundation (FS 98005). Dr Piepoli and Mr Scott are supported by the Wellcome Foundation. Professor Coats is supported by the Viscount Royston Trust.

1 Ho K, Anderson K, Kannel W, et al. Survival after the onset of congestive heart failure in Framingham heart study subjects. Circulation 1993;88:107-15.

2 Kupari M, Lindroos M, Iivanainen A, et al. Congestive heart failure in old age: prevalence, mechanisms and 4-year
prognosis in the Helsinki ageing study. F Intern Med 1997; 241:387-94. 
3 Garg R, Yusuf S. Epidemiology of congestive heart failure. In: Barnett DB, Pouler H, Francis GS, eds. Congestive cardiac failure - pathophysiology and treatment. New York: Marker 1993:9-25.

4 Mudge GH, Goldstein S, Addonizio LJ, et al. 24th Bethesda conference taskforce 3: heart transplantation: recipient guidelines/prioritization. F Am Coll Cardiol 1993;22:21-31.

5 Bruce RA, Blackman JR, Jones JW. Exercise testing in adult normal subjects and cardiac patients. Paediatrics 1963;32: $742-55$.

6 Clark A, Volterrani M, Swan JW, et al. Leg blood flow, metabolism and exercise capacity in chronic stable heart failure. Int 7 Cardiol 1996;55:127-35.

7 Collinson P. Of bombers, radiologists, and cardiologists: time to ROC. Heart 1998;80:215-17.

8 Wang R, Mouliswar M, Denman S, et al. Mortality of the institutionalized old-old hospitalized with congestive heart failure. Arch Intern Med 1998;158:2464-8.

9 Senni M, Tribouilloy C, Rodeheffer R, et al. Congestive heart failure in the community: a study of all incident cases in Olmsted County, Minnesota, in 1991. Circulation 1998; in Olmsted $2282-9$.

10 Cowburn P, Cleland J, Coats A, et al. Risk stratification in 998;19:696-710.

11 Pernenkil R, Vinson J, Shah A, et al. Course and prognosis in patients $\geqslant 70$ years of age with congestive heart failure and normal versus abnormal left ventricular ejection fraction. Am $\mathcal{F}$ Cardiol 1997;79:216-19.

12 Taffet G, Teasdale T, Bleyer A, et al. Survival of elderly men with congestive heart failure. Age Ageing 1992;21: 49-55.

13 Marburger C, Brubaker P, Pollock W, et al. Reproducibility of cardiopulmonary exercise testing in elderly patients with congestive heart failure. Am f Cardiol 1998;82:905-9.

14 Clark AL, Volterrani M, Swan JW, et al. The increased ventilatory response to exercise in chronic heart failure: relation to pulmonary pathology. Heart 1997;77: 138-46.

15 Chua TP, Ponikowski P, Harrington D, et al. Clinical correlates and prognostic significance of the ventilatory response
to exercise in chronic heart failure. $\mathcal{F} \mathrm{Am}$ Coll Cardiol 1997; to exercise in $1585-90$.

16 Uren NG, Davies SW, Agnew JE, et al. Reduction of mismatch of global ventilation and perfusion on exercise is related to exercise capacity in chronic heart failure. $B$ Heart f 1993;70:241-6.

17 Reindl I, Wernecke K-D, Opitz C, et al. Impaired ventilatory efficiency in chronic heart failure: possible role of pulmonary vasoconstriction. Am Heart f 1998;136:778-85

18 Buller NP, Poole-Wilson PA. Mechanism of the increased ventilatory response to exercise in patients with chronic heart failure. Br Heart f 1990;63:281-3.

19 Kleber FX, Reindl I, Werneke KD, et al. Dyspnea in heart failure. In: Wasserman K, ed. Exercise gas exchange in heart disease. Armonk, New York: Futura, 1995:95-108.

20 Chua TP, Clark AL, Amadi AA, et al. Relation between chemosensitivity and the ventilatory response to exercise in chronic heart failure. $\mathcal{F}$ Am Coll Cardiol 1996;27:650-7.

21 Mancini DM, Eisen H, Kussmaul W, et al. Value of peak exercise oxygen consumption for optimal timing of cardiac transplantation in ambulatory patients with heart failure. Circulation 1991;83:778-86.
22 Van den Broek S, Van Veldhuisen D, De Graeff P, et al. Comparison between New York Heart Association classification and peak oxygen consumption in the assessment of functional status and prognosis in patients with mild to moderate chronic congestive heart failure secondary to either ischaemic or idiopathic dilated cardiomyopathy. $\mathrm{Am}$ f Cardiol 1992;70:359-63.

23 Szlachcic J, Massie BM, Kramer BL, et al. Correlates and prognostic implication of exercise capacity in chronic congestive heart failure. Am f Cardiol 1985;55:1037-42.

24 Chomsky DB, Lang CC, Rayos GH, et al. Hemodynamic exercise testing. A valuable tool in the selection of cardiac transplantation candidates. Circulation 1996;94:3176-83.

25 Opasich C, Pinna GD, Bobbio M, et al. Peak exercise oxygen consumption in chronic heart failure: toward efficient use in the individual patient. $\mathcal{F ~ A m ~ C o l l ~ C a r d i o l ~ 1 9 9 8 ; 3 1 : 7 6 6 - 7 5 . ~}$

26 Harrington D, Anker SD, Chua TP, et al. Skeletal muscle function and its relation to exercise tolerance in chronic function and its relation to exercise tolerance in
heart failure. $f \mathrm{Am}$ Coll Cardiol. 1997;30:1758-64.

27 Yasaka Y, Yamabe H, Yokoyama M. Dependence of peak oxygen uptake on oxygen transport capacity in chronic heart failure: comparison of graded protocol and fixed proheart failure: comparison of graded p

28 Lipkin DP, Jones DA, Round JM, et al. Abnormalities of skeletal muscle in patients with chronic heart failure. Int $\mathcal{F}$ Cardiol 1988;18:187-95.

29 Anker SD, Ponikowski P, Varney S, et al. Wasting as independent risk factor for mortality in chronic heart failure. Lancet 1997;349:1050-3.

30 Adams KF Jr, Dunlap SH, Sueta CA, et al. Relation between gender, etiology and survival in patients with symptomatic heart failure. $\mathcal{F}$ Am Coll Cardiol 1996;28:1781-8.

31 Lee WH, Packer M. Prognostic importance of serum sodium concentration and its modification by convertingenzyme inhibition in patients with severe heart failure. Circulation 1986;73:257-67.

32 Parameshwar J, Keegan J, Sparrow J, et al. Predictors of prognosis in severe chronic heart failure. Am Heart f 1992; 123:421-6.

33 Wong M, Johnson G, Shabetai R, et al. Echocardiographic variables as prognostic indicators and therapeutic monitors in chronic congestive heart failure: Veterans Affairs cooperative studies V-HeFT I and II. Circulation 1993;87: V165-70

34 Batin PD, Wickens M, McEntegart D, et al. The importance of abnormalities of liver function tests in predicting mortality in chronic heart failure. Eur Heart $\mathcal{f}$ 1995;16: 1613-18.

35 Rickenbacher PR, Trindale PT, Haywood GA, et al. Transplant candidates with severe left ventricular dysfunction managed with medical treatment: characteristic and survival. F Am Coll Cardiol 1996;27:1192-7.

36 Coats AJ. The "muscle hypothesis" of chronic heart failure. $7 \mathrm{Mol}$ Cell Cardiol 1996;28:2255-62.

37 Willenheimer RB, Erhardt LR, Cline CMJ, et al. Prognostic significance of changes in left ventricular systolic function in elderly patients with congestive heart failure. Coron Artery Dis 1997;8:711-17.

38 Vasan RS, Larson MG, Benjamin EJ, et al. Congestive heart failure in subjects with normal versus reduced left ventricular ejection fraction. $f$ Am Coll Cardiol 1999;33. 1948-55. 